\title{
Sustainability method organizational and technological decisions in the construction of industrial complexes
}

\author{
Zinur Mukhametzyanov ${ }^{1 *}$, Pavel Oleinik ${ }^{2}$ \\ ${ }^{1}$ Ufa State Oil Technical University, 450062, Ufa, Russia, \\ ${ }^{2}$ Moscow State University of Civil Engineering, 129377, Moscow, Russia
}

\begin{abstract}
The task is to develop a mechanism for the implementation of organizational and technological solutions in the construction of industry complexes. The action of the mechanism is based on the relationship of the parameters of organizational and technological design and construction decisions, which increases the reliability of the implementation of decisions. A conceptual approach has been identified to solve the problem of improving the reliability of organizational and technological solutions in the construction of industry complexes. A model of the technology for erecting an object using estimates of technological dependencies is developed, which is the technological basis for making organizational and technological decisions. The algorithm for the implementation of organizational and technological solutions in the construction of industrial complexes is determined. The proposed mechanism provides an increase in the multiplicity of ways to select the optimal set of organizational and technological solutions due to a significant expansion of the zone of feasible solutions and thereby solves the problem of ensuring the stability and reliability of organizational and technological solutions in the construction of industrial complexes.
\end{abstract}

\section{Introduction}

The construction of industrial complexes for various purposes, characterized by high dynamism, i.e. the constant change in the forms, methods and conditions of work, the need to comply with the interests of the owner for the effective investment of investor capital in the construction of complexes is a complex and time-consuming task. Construction practice shows that for the construction of each new industry complex, it is necessary to study all possible options for the organization of work in order to compare them and select the most rational of them for specific production conditions.

In solving this problem, a conceptual approach is the development of methods to increase the reliability of organizational and technological decisions (OTD) [1-14].

* Corresponding author: zinur-1966@mail.ru 
Currently, the basis for the formation of OTD are the principles of building systems engineering [15-18]. In the framework of this area of research, Russian specialists have developed various ways to increase the reliability of OTD. These include the technique of reducing organizational and technological processes into indistinguishable groups of reliability, the development of methodological foundations for the design of organizational and technological processes that provide system-technical coordination of functional subsystems and information-analytical tasks in an information-computing environment, methods of increasing reliability with taking into account the risk in the design of the organization, technology and construction management in the face of uncertainty, "the project approach", taking into account market fundamentals of building design, the development of construction organization projects by integrating the flow with the principles of integrated mechanization and the use of kibernetic construction management systems. From foreign studies, methods based on a buffer management strategy, creating an integrated metric for quantifying the stability of interdependent systems, and ensuring the stability of processes in construction are distinguished.

Nevertheless, the experience of using the listed domestic and foreign developments shows that no effective result has been achieved in increasing the degree of reliability of OTD during the construction of industry-specific complexes. A generalized analysis allows you to combine the reasons for this result in the following groups:

1. justification and adoption of OTD is carried out without taking into account their relationship at the design and construction stages;

2. the substantiation and adoption of OTD is carried out on the basis of a rigid relationship of time, volume and resource parameters of the organizational and technological model, which leads to adjustment of parameters even with a minimum deviation of one of these parameters;

3. OTD are justified and accepted without taking into account the feedback between the technological and organizational subsystems.

To solve these problems, a new mechanism is proposed for the formation of the OTD system during the construction of industrial complexes, which eliminates the identified causes and ensures the achievement of stability and reliability of the OTD.

\section{Definition of approaches to solving the problem of achieving reliability organizational and technological solutions}

The construction of industry complexes, as an integral component of the modern construction industry, has its own objective laws that allow us to identify a study methodology, as well as determine ways to create models, and select decision-making algorithms for organizing the construction of complexes.

The industry complex is a multicomponent system, which is characterized by the following properties:

- the presence of several interconnected subsystems (enterprises, facilities), the construction of which is carried out independently from each other, but having a common completion date;

- the constancy of the purpose of the construction of subsystems (objects);

- the invariability of the structure, construction characteristics of subsystems (objects);

- the dynamism of the parameters of the construction of subsystems, which means the use of modeling not static parameters, but dynamic in the form of the pace of construction and installation operations (CIO); 
- the uncertainty of the external environment, in the context of which the construction of industry complexes occurs, which arises as a result of the impact of a multitude of poorly formalized criteria, including economic and social ones.

The distinguished properties make it possible to consider the process of building an industry complex as combining the construction of many objects that make up an industry complex. Then, when analyzing the sustainability of OTD during the construction of industry complexes, the task arises of studying the relationship between the characteristics of the system of organizational and technological solutions of individual objects (subsystems) and industry complexes in order to identify the continuity of organizational and technological solutions of design and construction.

Achieving the continuity of organizational and technological solutions when designing them as part of the projects for the organization of construction (PCO) of industrial complexes allows us to solve the problem of guaranteed implementation of construction schedules.

\section{Development of a method for ensuring the reliability of organizational and technological solutions in the construction of industry complexes.}

When developing organizational and technological solutions for the construction of modern industry complexes, the following features should be taken into account:

- a variety of types of construction models of industrial complexes;

- combination of construction and synchronization of commissioning of all the facilities that make up the complex, with the goal of putting the entire complex in commission by the planned completion date.

Since the construction of industrial complexes involves the construction of various facilities, one should expect many technologies for their construction. At the same time, it is necessary to take into account the fact that in order to solve the stated problem of ensuring the construction of complexes within the planned time frame, a system of organizational and technological decisions is needed that is unified for all the facilities that make up the complex, which is resistant to the factors inherent in construction and guarantees a certain probability of achieving the planned result. It is important to emphasize the unified properties so that the system of making organizational and technological decisions is based on a single platform and, thus, achieves efficiency and convenience in practical implementation.

In this regard, when developing a methodology for the adoption of OTD for the construction of industrial complexes that are resistant to the action of factors affecting the stability of the course of construction, the following restrictions should be taken into account:

- the relationship of the parameters of the OTD system for the construction of individual facilities and industry-specific complexes;

- the immutability of the policy deadlines for the construction of individual facilities that make up the industry complex. At the same time, a change in the course of construction of objects is allowed, leading ultimately to a decrease in the planned construction time;

- the construction period for the industry complex is long enough to consider the construction process as stationary;

- it is assumed that the intensity of the construction progress of the industrial complex is significant and regular, which allows us to consider the construction process as continuous;

- as the subsystems are taken the individual objects that make up the complex; 
- the system for the adoption of organizational and technological decisions of OTD during the construction of all objects (subsystems) that are part of the complex is formed according to a single principle and form a single platform on which OTD is implemented for the construction of the industry complex.

Reliable implementation of OTD projects for the organization of construction (PCO) of industrial complexes is ensured through an integrated system for the implementation of the OTD model of method statement (MS), built on the basis of the identified rules of technological interaction between the processes.

The basis of the OTD MS model is the model of the facility construction technology built using qualitative and quantitative estimates of technological dependencies between interrelated processes (Fig. 1).

A qualitative characteristic of technological relations between the construction processes determines the order of priority of work relative to each other and serves as the basis for establishing a certain order of interaction of construction processes used in the construction of an object.

A quantitative assessment of the technological interconnection of adjacent processes is the value of the minimum technologically necessary volume, which allows you to plan and organize the execution of the next technologically interconnected process at its beginning ( $\left.V_{(i, i+1)}^{H}\right)$ and end $\left(_{(i, i+1)}^{o}\right)$ (Fig. 1).

The model thus obtained is a sustainable technological basis for the adoption of OTD. The stability of such a model is achieved due to determinism throughout the production process at the facility for quantifying technological connections at the beginning and end of processes. Such determinism provides the construction of a "rigid" frame of the technological base model, which ensures compliance with the duration of work adopted in the PCO.

Further development of OTD based on this model is as follows.

OTDs are developed not for the entire construction schedule of the facility, the duration of which is laid down in the PCO, but for the schedule of work for a separate time period for each facility that is part of the complex, and this time period coincides with the construction period for which capital investments and CIO are allocated, adopted in the PCO calendar plan.

\begin{tabular}{|c|c|c|c|c|}
\hline № & Name of work item & $\begin{array}{l}\text { Beginning of } \\
\text { Jork 01.08.2016 }\end{array}$ & & $\begin{array}{l}\text { Completion of } \\
\text { work } \\
22.10 .2017\end{array}$ \\
\hline 1 & Metal fabrication & $0 \cdots$ & $=$ & \\
\hline 2 & $\begin{array}{l}\text { Mounting the bottom of the } \\
\text { outdoor tank }\end{array}$ & $V_{1,2}^{\text {n }}$ & \begin{tabular}{l|l}
$\boldsymbol{*}_{\boldsymbol{A}}$ & $V_{2,3}^{\circ}$ \\
\end{tabular} & \\
\hline 3 & $\begin{array}{l}\text { Mounting the bottom of the } \\
\text { inner tank }\end{array}$ & 4 & $\because \cdots \cdot 4$ & \\
\hline 4 & $\begin{array}{l}\text { Mounting the walls of the } \\
\text { inner and outer reservoir }\end{array}$ & & $\because 4$ & $V_{5.6}^{\circ}$ \\
\hline 5 & $\begin{array}{l}\text { Mounting the roof of the } \\
\text { inner tank }\end{array}$ & $V_{4,5}^{\mathrm{H}}$ & & \\
\hline 6 & $\begin{array}{l}\text { Mounting the roof of the } \\
\text { outdoor tank }\end{array}$ & $V_{4,6}^{\mathrm{H}}$ & & \\
\hline 7 & $\begin{array}{l}\text { Installation of hatches, pipes, } \\
\text { fittings, irrigation rings }\end{array}$ & $V_{9,10}^{\mathrm{H}}$ & & \\
\hline 8 & Resistance tank tests & & $V_{4,6}^{\mathrm{H}}$ & 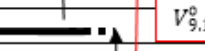 \\
\hline 9 & $\begin{array}{l}\text { Atmospheric corrosion } \\
\text { protection }\end{array}$ & $\cdots \cdots$ & & \\
\hline 10 & Thermal insulation & & & \\
\hline
\end{tabular}

Fig. 1. The model of technology for the construction of an object (isothermal tank) 
Moreover, specialized flows are divided into processes and these processes are interconnected according to the rules of technological interaction of processes. The main criterion for the dismemberment is the compliance of the estimated cost of the total set of processes with the financing schedule according to the PCO calendar plan within the framework of the accepted duration of work.

Based on the results of the division of work into processes, recommendations are developed for compiling a work schedule, linked to current standards and indicators such as: the minimum lag of the beginning of subsequent work from the beginning of the previous one and the minimum lag of the end of subsequent work from the end of the previous $\left(V_{(i, i+1)}^{o}, V_{(i, i+1)}^{H}\right)$; duration of each work item ${ }^{\left(t_{i, j}\right)}$; the minimum combination of work performed at the beginning and end of their implementation $\left(S_{(j, j+1)}^{H}, S_{(j, j+1)}^{o}\right)$; the amount of labor for each work item $\left(R^{h}(t)\right)$; the amount of material resources for each work item $\left(M_{\mu, \tau}\right)$ ( Fig. 2). But it is necessary to fulfill the following conditions related to taking into account the quantitative and qualitative characteristics between technologically related processes.

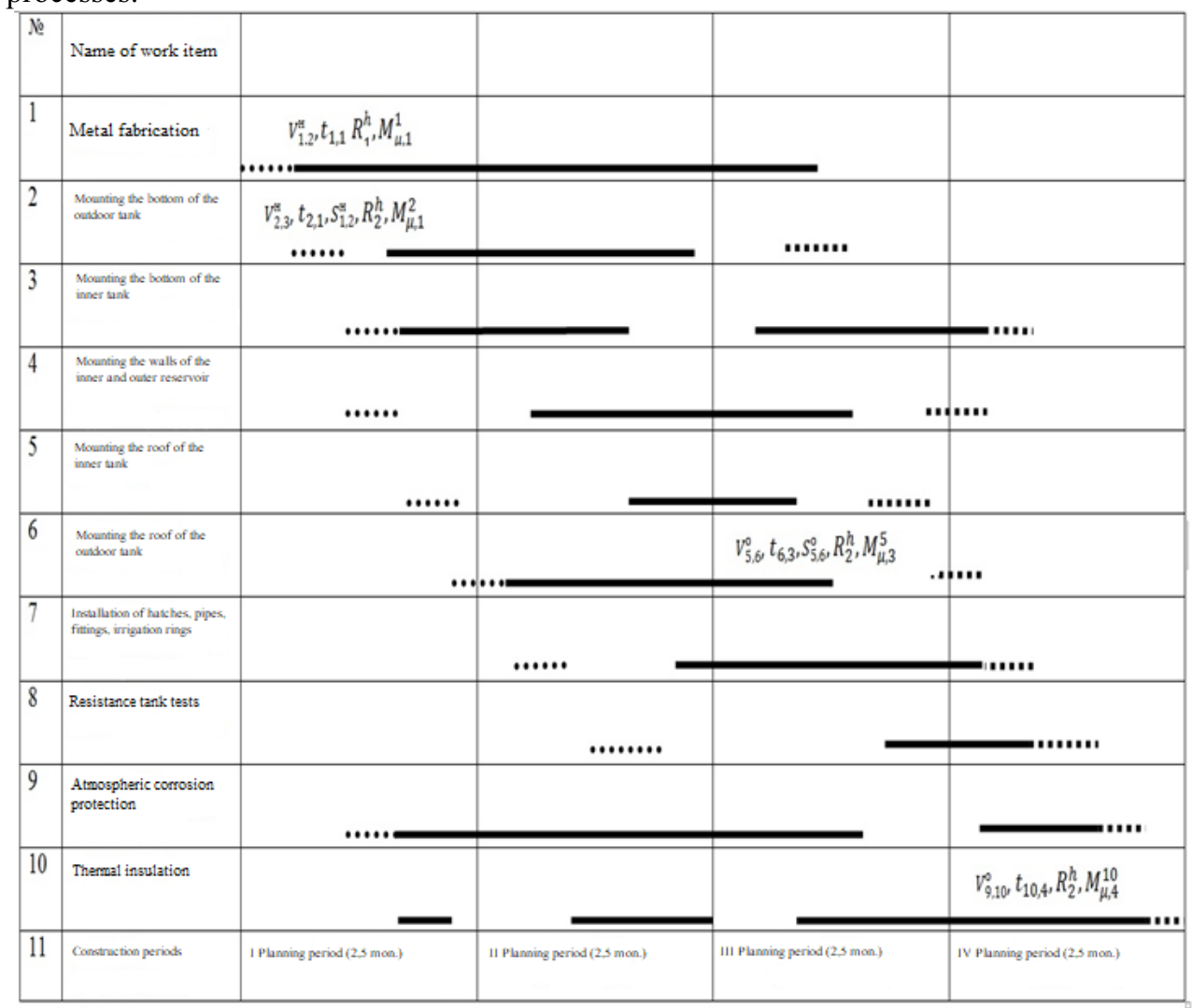

Fig. 2. An example of constructing a calendar schedule of work based on the model of technological connections

The first condition (restriction) that must be taken into account is introduced to calculate the necessary labor resources for the implementation of the planned volumes of processes in a certain planning period. 
Planning the necessary volume of the process $\mathrm{j}$ is possible subject to the following conditions:

$$
V_{j-k, i, \tau}+\sum_{\tau=1}^{\tau-1} V_{j-k, i} \geq V_{\min j, j-k, i}^{H}(\tau=1,2, \ldots \bar{\tau})
$$

where $V_{j-k, i, \tau}$ - the planned volume of the preceding process $(\mathrm{j}-\mathrm{k})$ at facility $\mathrm{i}$ in the planning period $\tau$, with which process $j$ is technologically connected; $\sum_{\tau=1}^{\tau-1} V_{j-k, i}$ - the amount of the previous process $(j-k)$ at facility $i$, completed (planned) in previous planning periods; $V_{\min j, j-k, i}^{H}$ - the minimum volume that must be completed (or planned to be completed) in the previous process $(j-k)$ for planning to be executed per unit volume of the process $j$.

The second condition is the restriction on the planned volume of the process $j$ in the interim planning period $\tau$. In accordance with this restriction, the planned volume should not exceed the volume, the fulfillment of which is possible at the maximum saturation of the process front with resources such as capacity:

$$
V_{i, j, \tau} \leq V_{\max i, j, \tau}
$$

where $V_{\max i, j, \tau}$ - the volume of the process that can be performed at maximum saturation with labor resources.

The third condition is the restriction or the need to reinforce the work planned in a certain temporary planning period with material resources:

$$
M_{\mu, \tau}=\sum_{i=1}^{n} \sum_{j=1}^{m} n_{\mu, j} V_{j, i, \tau}^{p l}
$$

where $M_{\mu, \tau}$ - the amount of material resources of type $\mu$, which may be in the construction organization during the period $n_{\mu, j}$ - rate of material consumption per unit of work $j ; V_{j, i, \tau}^{p l}-$ the volume of work planned to be performed at the facility during a certain planning period in physical measurements.

Then the necessary resources of the type of capacity necessary to carry out the processes planned for a certain planning period are formed according to the following formula:

$$
R^{h}=\sum_{j=1}^{m} \sum_{i=1}^{n} \frac{C_{j}^{u n} V_{j, i, \tau}^{p l}}{g_{\tau}^{h}}=R_{\text {rat }}^{h}
$$

where $C_{j}^{u n}$ - process unit cost; $V_{j, i, \tau}^{p l}$ - the volume of processes planned to be completed at the facility during a certain planning period in physical measurements; $i$ - plan period number, ${ }^{i}=1,2, \ldots n ; j$ - process number in the planning period, $j=1,2, \ldots m{ }{ }_{\tau}$ - plan period number; $g_{t}^{h}$-daily value output by industrial worker job $h$.

The resulting ratio indicates $i=1,2, \ldots n$; the amount of necessary resources such as capacity for the implementation of the entire planned volume of processes in a certain planning period as part of the implementation of the financing schedule according to the PCO calendar plan.

\section{Conclusion}


The developed mechanism for the adoption of OTD during the construction of industry complexes allows us to solve the problems characteristic of existing approaches to increasing the reliability of OTD, by ensuring the conditions for the continuity of organizational and technological solutions of PCO and organizational and technological solutions of MS, eliminating their divergence. Such conditions are achieved:

- Dividing the specialized flows into processes whose estimated cost of a common set corresponds to the financing schedule according to the PCO calendar plan within the framework of the accepted duration of the work.

- The adoption of the OTD model of the technology for the construction of buildings and structures, which is a sustainable technological basis. The stability of such a model is achieved due to determinism throughout the production process at the facility for the quantitative assessment of technological connections at the beginning and end of processes.

- The development of the OTD model is not for the entire construction schedule of the facility, the duration of which is laid down in the PCO, but for the schedule of work for a separate time period for each facility that is part of the complex, and this time period coincides with the construction period for which capital investments and construction and installation works are allocated PCO schedule.

Scientific novelty lies in the author personally identifying a new approach to ensuring the stability and reliability of OTD during the construction of industry complexes, which consists in constructing the OTD on the basis of modeling the technology of construction of objects through the use of qualitative and quantitative assessments of technological interconnections between processes.

The practical significance of the developed mechanism consists in the possibility of using any project in conjunction with the current regulatory documents for any project taking into account the specific production conditions of the construction site, work schedules and processes in technological maps, etc.

\section{References}

1. Z.R. Mukhametzyanov, Modeling of construction technology of objects on the basis of technological interaction of works, IOP Conference Series: Materials Science and Engineering, 451 (2018).

2. Z.R. Mukhametzyanov, E.V. Gusev, Numerical modeling of technological volume between adjacent construction processes, IOP Conference Series: Materials Science and Engineering, 451 (2018).

3. Z.R. Mukhametzyanov, A.V. Rubtsov, A.S. Valiev, Estimation of Influence Character of Installation Loads on Degree of Corrosive Wear of Column Apparatus, Proceedings of the 4th International Conference on Industrial Engineering. ICIE. Lecture Notes in Mechanical Engineering. Springer, Cham, 1999 (2018).

4. Z.R. Mukhametzyanov, R.V. Razyapov, Mechanism of development of organizational solutions based on a technological interaction between construction works and processes, Russian Journal of Building Construction and Architecture, 3 (39) 85 (2018).

5. E.V. Gusev, Z.R. Mukhametzyanov, R.V. Razyapov, Technique for Determination of Rational Boundaries in Combining Construction and Installation Processes Based on Quantitative Estimation of Technological Connections, IOP Conference Series: Materials Science and Engineering (MSE), 1 (2017).

6. P. Kagan, T. Barabanova, The Formal Language for Describing Technological Processes in Construction, Computing in Civil and Building Engineering, 2232 (2014). 
7. P. Kagan, The engineering communication networks - the issues of use of standards for the infor-mation representation in design, construction and operation, Procedia Engineering, 25, 261 (2016).

8. P. Kagan, Management, mechanization and automation of work in the construction of high-rise buildings, MATEC Web of Conferences, 170 (2018).

9. A. Salosin, O. Gamayunova, A. Mottaeva, EMMFT 2019, IOP Publishing, 1614 012028 (2020). doi:10.1088/1742-6596/1614/1/012028

10. P. Kagan, A. Naumova, Y. Vilman, The Problems of project management software implementation in construction corporations, Web of Conferences, 73 (2016).

11. V.V. Granev, E.N. Kodysh, Development and updating of normative documents on design and construction of industrial and civil buildings, Industrial and civil construction, 79 (2014).

12. P.P. Oleinik, A. Yurgaytis, Optimization of the annal construction program solution, Mames Web of conferences 117, Theoretical Foundation of Civil Engineering, 00130 XXVIR-S-P Seminar, 8 (2017).

13. P.P. Oleinik, T.K. Kuzmina, Modelling the Redaction Project Making Duration, Mames Web of conferences 117, Theoretical Foundation of Civil Engineering, 00129. XXVIR-S-P Seminar, 5 (2017).

14. P.P. Oleinik, Organizational and technological support of construction of modern industrial enterprises, Mechanization of construction, 7 (2017).

15. P.P. Oleinik, Method for creating a work management plan of a construction company, International journal of Construction Management, 1 (2017).

16. M.A. Farag, Bridge between increasing reliability and reducing variability in construction work flow: A fuzzy-based sizing buffer model, Journal of Advanced Management Science, 2, 56 (2014).

17. Z.R. Mukhametzyanov, R.V. Razyapov, Classification of combinations of technologically interconnected construction processes used in the construction of the object, Industrial and civil construction, 10, 72 (2017).

18. Z.R. Mukhametzyanov, Qualitative and quantitative criteria of the efficiency assessment of the construction processes, Monograph (Rossiyskoy inzhenernoy akademii, Moscow, 2016). 